\title{
The role of stellar radial motions in shaping galaxy surface brightness profiles
}

\author{
T. Ruiz-Lara ${ }^{1,2,3,4}$, C. G. Few ${ }^{5,6}$, E. Florido ${ }^{3,4}$, B. K. Gibson ${ }^{5,6}$, I. Pérez ${ }^{3,4}$, and P. Sánchez-Blázquez ${ }^{7}$ \\ 1 Instituto de Astrofísica de Canarias, Calle Vía Láctea s/n, 38205 La Laguna, Tenerife, Spain \\ e-mail: tomasruizlara@gmail.com \\ 2 Departamento de Astrofísica, Universidad de La Laguna, 38200 La Laguna, Tenerife, Spain \\ 3 Departamento de Física Teórica y del Cosmos, Universidad de Granada, Campus de Fuentenueva, 18071 Granada, Spain \\ 4 Instituto Carlos I de Física Teórica y computacional, Universidad de Granada, 18071 Granada, Spain \\ 5 E.A. Milne Centre for Astrophysics, University of Hull, Hull HU6 7RX, UK \\ 6 Joint Institute for Nuclear Astrophysics, Center for the Evolution of the Elements (JINA-CEE), Indiana 46556, USA \\ 7 Departamento de Física Teórica, Universidad Autónoma de Madrid, 28049 Cantoblanco, Spain
}

Received 1 July 2017 / Accepted 20 September 2017

\begin{abstract}
Aims. The physics driving features such as breaks observed in galaxy surface brightness (SB) profiles remains contentious. Here, we assess the importance of stellar radial motions in shaping their characteristics.

Methods. We use the simulated Milky Way-mass cosmological discs from the Ramses Disc Environment Study (RaDES) to characterise the radial redistribution of stars in galaxies displaying type-I (pure exponentials), II (downbending), and III (upbending) SB profiles. We compare radial profiles of the mass fractions and the velocity dispersions of different sub-populations of stars according to their birth and current location.

Results. Radial redistribution of stars is important in all galaxies regardless of their light profiles. Type-II breaks seem to be a consequence of the combined effects of outward-moving and accreted stars. The former produce shallower inner profiles (lack of stars in the inner disc) and accumulate material around the break radius and beyond, strengthening the break; the latter can weaken or even convert the break into a pure exponential. Further accretion from satellites can concentrate material in the outermost parts, leading to type-III breaks that can coexist with type-II breaks, but situated further out. Type-III galaxies would be the result of an important radial redistribution of material throughout the entire disc, as well as a concentration of accreted material in the outskirts. In addition, type-III galaxies display the most efficient radial redistribution and the largest number of accreted stars, followed by type-I and II systems, suggesting that type-I galaxies may be an intermediate case between types II and III. In general, the velocity dispersion profiles of all galaxies tend to flatten or even increase around the locations where the breaks are found. The age and metallicity profiles are also affected, exhibiting different inner gradients depending on their SB profile, being steeper in the case of type-II systems (as found observationally). The steep type-II profiles might be inherent to their formation rather than acquired via radial redistribution.
\end{abstract}

Key words. galaxies: evolution - galaxies: spiral - galaxies: stellar content - galaxies: formation - galaxies: structure methods: numerical

\section{Introduction}

The observed properties of spiral galaxies are the outcome of complex, non-linear, and inter-related, formation and evolution processes. According to the current paradigm, after the assembly of large galaxies via a number of high-redshift minor mergers, a period of secular evolution follows. In parallel with the latter, non-axisymmetric structures such as bars or spiral arms may be formed (e.g. White \& Rees 1978; Abadi et al. 2003) with the ability to redistribute material (Sellwood \& Binney 2002; Roškar et al. 2008b,a; Minchev \& Famaey 2010) across the entire galaxy. Simultaneous to this secular evolution, satellite accretion may continue to influence the characteristics of the host (Younger et al. 2007; Bird et al. 2012; Ruiz-Lara et al. 2016a), particularly those found in the outermost regions.

A growing number of theoretical and observational works are focused on analysing the characteristics of these outer parts of spiral galaxies (e.g. Roškar et al. 2008b,a; Sánchez-Blázquez et al. 2009; Minchev et al. 2012; Bakos et al. 2008; Yoachim et al. 2012; Ruiz-Lara et al. 2016b). The lower surface densities (and, hence, gravitational effects) and the longer dynamical times of stars populating those regions make the study of the outskirts of disc galaxies a unique place to test current galaxy formation and evolution models. One of the defining metrics of these outer regions is the presence, or lack thereof, of deviations from a pure exponential surface brightness (SB) profile (Patterson 1940; Freeman 1970; Bland-Hawthorn et al. 2005; van der Kruit 1987; Pohlen et al. 2002; Pérez 2004; Trujillo \& Pohlen 2005; Erwin et al. 2005; Florido et al. 2006; Martín-Navarro et al. 2012). While some galaxies possess an essentially pure exponential SB profile into the outer parts (type I, continuation of the inner behaviour), others show a deficiency (type II) or excess of light (type III) that can be characterised by two exponentials (Pohlen \& Trujillo 2006). The drivers behind these different profiles remain unclear, although changes in the ages of stellar populations, the effect of radial migration, and satellite accretion have all been proposed (Younger et al. 2007; Roškar et al. 2008b; Sánchez-Blázquez et al. 2009; Martínez-Serrano et al. 2009; Minchev et al. 2012).

In the last decade a clear link between an outer upturn ("U-shape") in the age profiles and type-II galaxies was found via observations and theory, with the age upturn causing the 
lack of light in the outer regions (e.g. Bakos et al. 2008; Roškar et al. 2008b; Sánchez-Blázquez et al. 2009; Martínez-Serrano et al. 2009; Yoachim et al. 2012). Conversely, in Ruiz-Lara et al. (2016b), analysing spectroscopic information from the Calar Alto Legacy Integral Field Area ${ }^{1}$ (CALIFA; Sánchez et al. 2012) survey, we found that "U-shape" age profiles are found for both type-I and II galaxies (similar to that found by Roediger et al. 2012, from photometric data). As a consequence, we suggested that the mechanisms causing the light distributions and the stellar-age profiles might not be coupled. In the same vein, Ruiz-Lara et al. (2016a) showed that all galaxies in the Ramses Disc Environment Study (RaDES; Few et al. 2012) simulation suite show "U-shape" stellar age profiles, regardless of their light distribution. Other observational studies have also found the presence of old stellar populations in the outer parts of nearby spiral galaxies (e.g. Gogarten et al. 2010; Bernard et al. 2012, 2015). All these works seem to indicate that a change in the stellar population age is not necessarily the main agent shaping the observed light profiles.

Other studies have concentrated on the role of satellite bombardment in shaping light profiles (e.g. Younger et al. 2007; Quillen et al. 2009; Bird et al. 2012). For instance, Younger et al. (2007) show that minor mergers can cause type-III SB profiles by concentrating mass in the inner regions or expanding the outer discs. However, most of the effort to understand the occurrence of the different SB profiles using simulations has been focused on analysing the effect of stellar radial motions/redistribution (Roškar et al. 2008b; Sánchez-Blázquez et al. 2009; Martínez-Serrano et al. 2009; Minchev et al. 2012; Grand et al. 2016; Sánchez-Menguiano et al. 2016).

Sánchez-Blázquez et al. (2009) found that downbending light profiles (type II) arise (together with "U-shape" age profiles) from the combined effect of i) an abrupt change in the radial star formation profile due to a change in the gas volume density profile linked to a warped disc (causing the truncation in the light profile) and ii) radial migration of stars formed in the inner parts towards positions located beyond the break radius. The authors claim that it is the first effect (flaring of the disc) which drives the U-shape age and downbending light profiles, while the second effect modifies the final shape of such profiles. However, they also speculate that different SB profiles might arise as a consequence of differences in the efficiency of both processes. In this way, if the distribution of gas volume density changes smoothly with galactocentric distance and the outwards radial redistribution of material is more efficient than in a type-II galaxy, one might obtain a type-III profile (upbending light profile). The same conditions might be applicable to pure exponential discs if the radial redistribution of material is less efficient than in type-III systems. Indeed, U-shaped age profiles can be a natural outcome in analytical models of pure exponentials with radially varying gas infall prescriptions, even in the complete absence of radial stellar (or gas) motions (Gibson et al. 2013). In a similar line of reasoning, Ruiz-Lara et al. (2017) recently found different stellar age and metallicity inner gradients for galaxies displaying type-I, II, and III SB profiles. They interpret those results as the outcome of a gradual increase in the radial redistribution efficiency from type-II to type-I and type-III galaxies $^{2}$.

Recently, several studies have tried to shed further light on the effect of radial redistribution of material in shaping SB profiles. Herpich et al. $(2015,2017)$ investigated the role of the halo

\footnotetext{
http://califa.caha.es/

An interpretation, admittedly, lacking a theoretical framework.
}

spin parameter $(\lambda)$ in shaping the outer SB profiles by analysing a set of controlled simulations of isolated galaxies. They found a clear transition from type-III systems displaying low spin parameters to type-II galaxies showing higher values with type-I discs having intermediate values $(\lambda \sim 0.035)$. In particular, they suggested that orbital resonances with a strong central bar, coupled with the low initial halo spin, can produce stellar migration which leads to upbending SB profiles. According to this work, stars populating these outer regions present high radial velocity dispersions and a lower degree of rotation than expected in disc-like systems. Minchev et al. (2012) found that after 2 Gyr of smooth, in-plane gas accretion, galaxies displaying typical type-II profiles acquire light upbendings in the outermost regions. This results in galaxies displaying a combination of an inner type-II and an outer type-III profile with the latter being populated by stars that also present high velocity dispersions. Borlaff et al. (2016) revisit the idea that flares in galaxies can give rise to discs following a type-II SB distribution. In Elmegreen \& Struck (2016), the authors present numerical experiments on the effect of stochastic scattering of random particles shaping single exponentials discs. They also report that type-II and III profiles could be found if a difference in the scattering bias for the inner and outer regions exists.

In this work, we make use of the RaDES suite of simulated galaxies to investigate two of the processes that can drive the different types of SB profiles: the effect of radial motions of stars and satellite accretion. In Sect. 2, we outline the simulations employed and characterise the associated galaxies. The main results and discussion are given in Sects. 3 and 4; our conclusions are presented in Sect. 5.

\section{Simulations and sample of galaxies}

The RaDES galaxies are simulated using the adaptive mesh refinement code Ramses (Teyssier 2002) tracking dark matter, stars, and gas on cosmological scales. The hydrodynamical evolution of gas uses a refining grid such that the resolution of the grid evolves to follow overdensities, reaching a peak resolution of $436 \mathrm{pc}$ (16 levels of refinement). In order to prevent numerical collapse, a polytropic equation of state is used for dense gas. If gas is more dense than $0.1 \mathrm{~cm}^{-3}$ star formation occurs at a rate given by $\dot{\rho}=-\rho / t_{\star}$, where $t_{\star}=t_{0}\left(\rho / \rho_{0}\right)^{-1 / 2}$ with $t_{0}=8 \mathrm{Gyr}$. Stellar feedback is delayed to occur $10^{7} \mathrm{yr}$ after star formation whereupon it distributes kinetic energy, mass, and metals to the gas within a two-grid-cell-radius sphere. The mass fraction of stellar particles that explode as supernovae $(\mathrm{SNe})$ is $10 \%$, with each SN providing $10^{51}$ erg of energy, and the $10 \%$ of non-metals ( $\mathrm{H}$ and $\mathrm{He}$ ) are converted to metals. The cosmological parameters employed in generating these realisations were: $H_{0}=70 \mathrm{~km} \mathrm{~s}^{-1} \mathrm{Mpc}^{-1}, \Omega_{\mathrm{m}}=0.28, \Omega_{\Lambda}=0.72, \Omega_{\mathrm{b}}=0.045$, and $\sigma_{8}=0.8^{3}$. Two different box-volume sizes of $20 \mathrm{~h}^{-1} \mathrm{Mpc}$ and $24 h^{-1} \mathrm{Mpc}$ were used. The mass resolution of dark matter particles was either $5.5 \times 10^{6} M_{\odot}$ or $9.5 \times 10^{6} M_{\odot}$, respectively, for each of the aforementioned two volumes. Further details of the halo selection process and the simulation parameters may be found in Few et al. (2012).

The RaDES suite is a powerful tool in assessing the effect of radial motions (including satellite accretion) in shaping SB profiles. In Few et al. (2012), we showed that these

\footnotetext{
3 Being $H_{0}$ the Hubble constant, $\Omega_{\mathrm{m}}$ the fraction of total matter, $\Omega_{\Lambda}$ the fraction of the dark energy, and $\sigma_{8}$ the strength of the primordial density fluctuations.
} 
Table 1. Main disc properties for the RaDES galaxies from the analysis of their $r$-SDSS band light distribution.

\begin{tabular}{lrrrrr}
\hline \hline Galaxy & SB type & $\begin{array}{r}h_{\text {in }} \\
(\mathrm{kpc})\end{array}$ & $\begin{array}{r}h_{\text {out }} \\
(\mathrm{kpc})\end{array}$ & $\begin{array}{r}R_{\text {break }} \\
(\mathrm{kpc})\end{array}$ & $\begin{array}{r}\beta \\
(\mathrm{kpc})\end{array}$ \\
\hline Apollo & II & 2.34 & 1.39 & 4.96 & 0.95 \\
Artemis & III & 0.79 & 5.87 & 5.30 & -5.08 \\
Atlas & II & 4.39 & 2.47 & 7.43 & 1.92 \\
Ben & II & 5.28 & 3.36 & 12.18 & 1.93 \\
Castor & II & 5.70 & 0.96 & 5.35 & 4.74 \\
Daphne & III & 1.26 & 3.84 & 10.08 & -2.58 \\
Eos & III & 2.95 & 8.55 & 18.32 & -5.60 \\
Helios & III & 1.93 & 7.76 & 11.49 & -5.83 \\
Hyperion & II & 4.31 & 2.77 & 14.97 & 1.54 \\
Krios & III & 2.62 & 8.45 & 16.11 & -5.83 \\
Leia & III & 3.81 & 7.48 & 21.21 & -3.67 \\
Leto & III & 0.99 & 4.07 & 6.59 & -3.08 \\
Luke & I & 5.78 & - & - & 0.00 \\
Oceanus & II & 8.08 & 4.18 & 23.17 & 3.90 \\
Pollux & III & 1.25 & 4.03 & 8.61 & -2.78 \\
Selene & II & 5.68 & 2.01 & 11.98 & 3.66 \\
Tethys & II & 4.43 & 2.12 & 9.82 & 2.31 \\
Tyndareus & III & 1.33 & 3.39 & 7.12 & -2.06 \\
Zeus & I & 0.92 & - & - & 0.00 \\
\hline
\end{tabular}

Notes. First column: Galaxy name. Second column: surface brightness type according to the Pohlen \& Trujillo (2006) classification. Third column: Inner disc scale-length in kpc. Fourth column: outer disc scalelength in kpc. Fifth column: break radius in kpc. Sixth column: strength of the break $(\beta)$ defined as $h_{\text {in }}-h_{\text {out }}$ in kpc.

Milky Way-mass, disc-dominated galaxies, possess characteristics resembling those of observed systems, such as metallicity gradients, matter content (total, dark, stellar, baryonic, and gaseous mass), and rotation curves (see also Pilkington et al. 2012). Unlike other comparable simulated cosmological samples, the RaDES galaxies are somewhat unique in possessing the full range of SB profiles (I, II, and III; Ruiz-Lara et al. 2016a).

\subsection{Characterisation of the RaDES galaxies light distribution}

The light profiles that we analyse in this work have been previously shown in Ruiz-Lara et al. (2016a). The radial profiles were computed by azimuthally averaging the light distribution from the mock images presented in (Few et al. 2012). These images were produced using the SUNRISE (Jonsson 2006) code mimicking the SDSS bandpasses. SUNRISE uses the stellar and gaseous distributions, as well as spectral energy distributions (SEDs) for each composite stellar particle, drawn from the Starburst99 stellar population models (Leitherer et al. 1999), in order to generate the bandpass-dependent mock images. The SB profiles computed in this way were then fitted with the function presented in (Erwin et al. 2008, Eqs. (5) and (6)) with a broken exponential profile implemented. This allows us to characterise the light distribution in these simulated galaxies in a similar fashion as usually done with observed photometric data (see Table 1). In the following analysis we concentrate on the results using the SDSS $r$-band light profiles, although identical results are found with the other two filters analysed in Ruiz-Lara et al. (2016a) (SDSS $g$ and $i$-bands).

A visual morphological classification from the mock images of these simulated galaxies suggests that the RaDES systems are mainly late-type disc galaxies. Thus, we have decided to compare these results with those found in Pohlen \& Trujillo (2006) analysing one-dimensional SB profiles of a sample of 98 latetype spiral galaxies in a similar way to in this work to ensure that these simulated galaxies display realistic light distributions. Table 1 shows the values of the main parameters describing the light profiles for all the RaDES galaxies. The average values of the inner disc scale-length $\left(h_{\text {in }}\right)$ that we obtain for the RaDES galaxies for type-I, II, and III galaxies are $\sim 3.3 \pm 1.2,5.0 \pm 0.8$, and $1.9 \pm 0.5 \mathrm{kpc}$, respectively. These values are in agreement (within errors) with those found in Pohlen \& Trujillo (2006) $(2.8 \pm 0.8,3.8 \pm 1.2$, and $1.9 \pm 0.6 \mathrm{kpc}$ for type-I, II, and III disc galaxies). Similar claims can be outlined regarding the position of the break. The RaDES type-II and III galaxies present values of $R_{\text {break }} / h_{\text {in }}$ of $2.2 \pm 0.4$ and $6.4 \pm 0.4$, respectively, while the values from the Pohlen \& Trujillo (2006) work are $1.9 \pm 0.6$ and $4.9 \pm 0.6$. Finally, we also find a good agreement if we consider the outer-disc scale-length $\left(h_{\text {out }}\right)$. The values of $h_{\text {out }}$ for the RaDES type-II and III galaxies are $2.4 \pm 0.5$ and $5.9 \pm 1.0 \mathrm{kpc}$, respectively, while for the Pohlen \& Trujillo (2006) sample those values are $2.1 \pm 0.9$ and $3.6 \pm 1.2 \mathrm{kpc}$, respectively. Greater differences are found for the $h_{\text {out }}$ and $R_{\text {break }} / h_{\text {in }}$ values for the type-III galaxies. These discrepancies might arise as a consequence of the well known "angular momentum problem" (e.g. Steinmetz \& Navarro 2002) causing the over-production of the spheroid component due to enhanced star formation at early epochs in cosmological simulations. Thus, we can claim that, although there are some small discrepancies with the typical observed SB profiles (especially for type-III galaxies), the light distributions in the RaDES set of galaxies are quite realistic and consistent with observations.

In order to establish a continuous parameter to characterise these SB profiles, from downbending to upbending (with pure exponentials as intermediate cases), we define the strength of the break $(\beta)$ as $h_{\text {in }}-h_{\text {out }}$. These $\beta$ values are also shown in Table 1 . We must highlight the continuity of this parameter, with the RaDES set of galaxies being comprised by galaxies presenting weak and strong type-II and III breaks as well as pure exponentials. We should note that all RaDES type-II galaxies also show an outer type-III break whose origin is also analysed in the following section (see Ruiz-Lara et al. 2016a). These type-II+type-III combined profiles have also been found in Minchev et al. (2012) as a consequence of in-plane gas accretion. Despite the existence of this secondary break, we will focus our SB characterisation on the $\beta$ parameter of the break located closer to the centre (with $\beta=0$ for type-I galaxies, negative for type-III galaxies, and positive for type-II galaxies).

In the following we name each analysed system according to the name given in Few et al. (2012). For further information on each particular galaxy we refer the reader to Few et al. (2012), Ruiz-Lara et al. (2016a), and Table 1.

\section{Role of radial redistribution in shaping SB profiles}

Several works seem to suggest that the role of radial redistribution of stars could be a decisive agent in shaping the SB profiles of spiral galaxies (Roškar et al. 2008b; Martínez-Serrano et al. 2009; Sánchez-Blázquez et al. 2009). In this study we take advantage of the fact that the RaDES sample presents realistic type-I, II, and III galaxies to assess if different radial redistribution patterns might give rise to the different observed SB profiles. To this aim, we characterise the radial redistribution of stars in the whole RaDES sample with SB profiles ranging from strong 

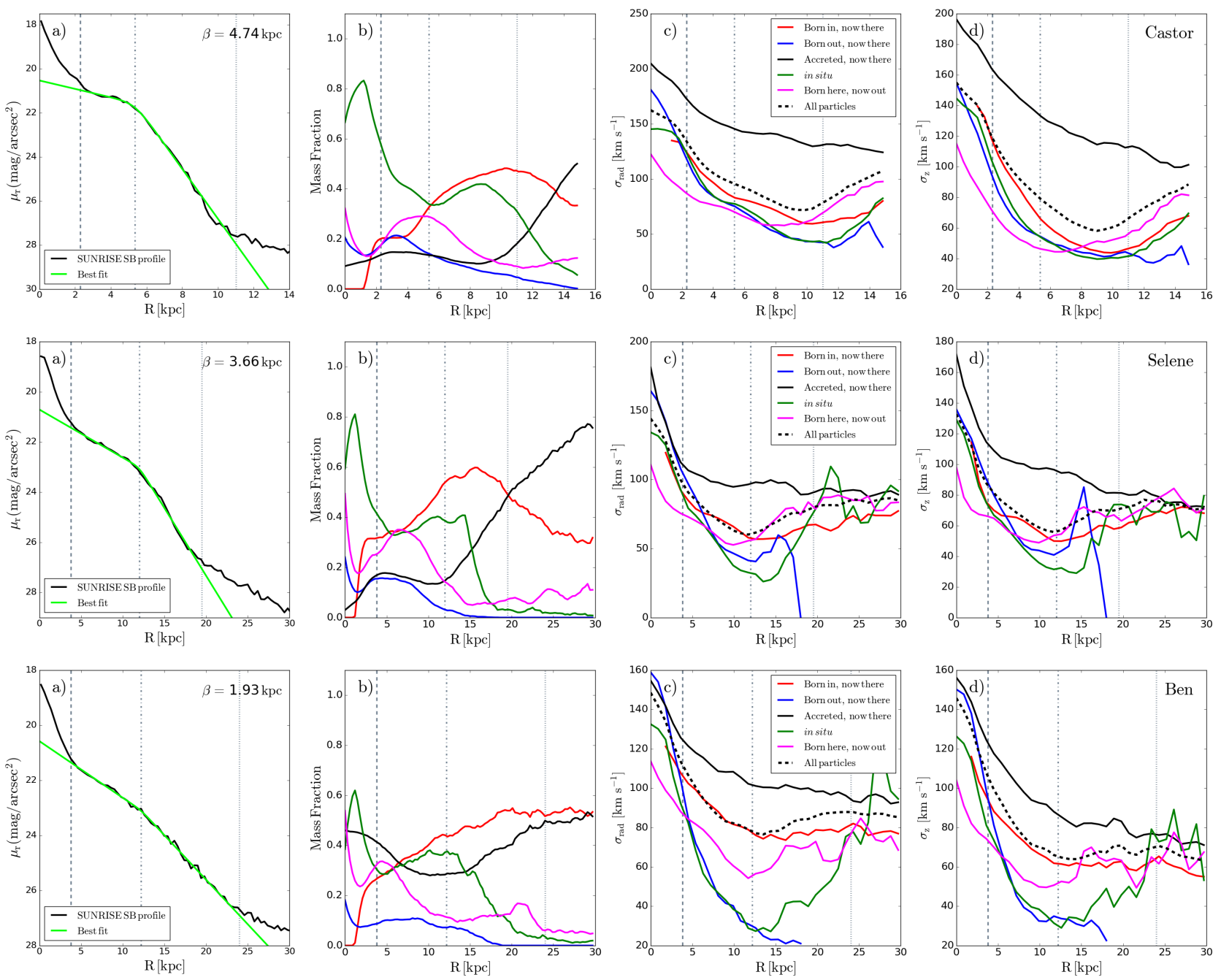

Fig. 1. Characterisation of the light, radial redistribution, and velocity dispersion profiles of the RaDES galaxies. Panel a: surface Brightness profiles in SDSS $r$ band for three type-II galaxies with different break strengths (Castor, Selene, and Ben). The black solid line represents the simulated (SUNRISE) SB profile while the light-green solid line is the best fit of the disc. Afterwards, from left to right: panel $b$ : radial redistribution of the stellar mass fraction; panel $c$ : radial velocity dispersion profiles; and panel $d$ : vertical velocity dispersion profiles for different subpopulations of stars. The different stellar subpopulations analysed include, for these three galaxies, stars not experiencing radial motions (green), those born at lower galactocentric distances and now found there (red), those born at higher galactocentric distances and now found there (blue), those born at that radius and currently located at a larger radius (magenta), and those coming from satellites (black). We have also included the radial and vertical velocity dispersion profiles considering all star particles in the simulation (black dashed lines). $\beta$, defined as $h_{\text {in }}-h_{\text {out }}$, is indicated as an inset. The vertical lines in all panels are located at the end of the bulge-dominated region $\left(\mu_{\text {model }}-\mu_{\text {observed }}<0.1\right.$ mag arcsec $^{-1}$, grey dashed line), the type-II break (grey dotted-dashed line), and the type-III break (grey dotted line). See text for further details.

type-II breaks $(\beta \sim 5$, e.g. Castor) to strong type-III breaks $(\beta \sim-6$, e.g. Helios). Such characterisation is based on the comparison of radial profiles of the mass fraction of stars with some specific characteristics according to their birth and current locations (see Figs. 1 and 2).

On the left-hand panel $(a)$ of Figs. 1 and 2 we show the SB profiles of six representative examples of galaxies displaying different SB profile types and break strengths. The second $(b)$ panel of the same figures shows the radial redistribution of stars in those galaxies; to that aim, this panel shows the radial profiles of the mass fraction of different stellar sub-populations according to their birth and current locations. The different sub-populations under analysis are: i) stars that are currently located at given galactocentric distances (denoted by $R$ ) coming from the inner parts (outward-moving stars, red line); ii) stars that are currently located at different galactocentric distances $(R)$ coming from the outer parts (inward-moving stars, blue line); iii) "in situ" stars (stars that have not moved from their birth place, green line); iv) stars born there and now located at a larger radius (magenta line); and v) accreted stars (black line). Although some of the above sub-population definitions are self-explanatory, "in situ" and accreted star require precise definition that could potentially affect our results. We define accreted stars as those whose $R_{\text {birth }}>20 \mathrm{kpc}$ and $\left|z_{\text {birth }}\right|>3 \mathrm{kpc}$ (following Ruiz-Lara et al. 2016a). We consider "in situ" stars as those whose absolute value of $R_{\text {birth }}-R_{\text {current }}$ is less than 0.2 disc scale-lengths (see Sect. 2.1). In the subsequent analysis we distinguish not only among galaxies with different SB profiles (I, II, or III) but also consider the fact that RaDES type-II galaxies have a more external upbending profile (type-II breaks denoted by the grey dotteddashed vertical lines while type-III breaks by the grey dotted vertical lines).

According to Figs. 1 and 2 we can claim that all galaxies have similar characteristics (regardless of their SB profile) in 

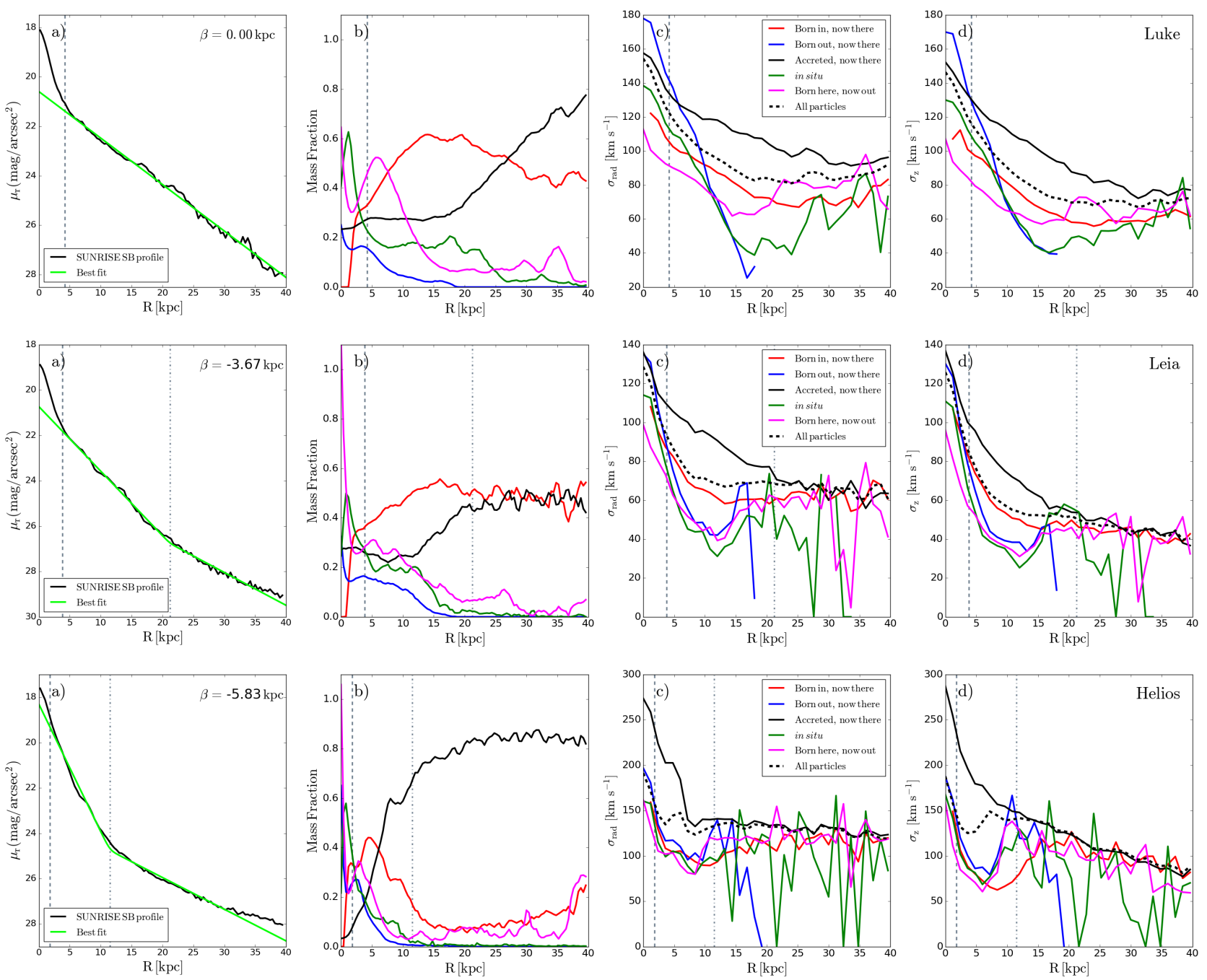

Fig. 2. Same as Fig. 1 but for one type-I and two type-III galaxies (with different break strengths), Luke, Leia, and Helios.

the bulge region ${ }^{4}$, delimited by the black vertical dashed line. Roughly speaking (and valid for most of the systems), the very centre is characterised by stars coming from regions at larger radius (blue line) but the amount of stars born in the very centre that have moved outward (magenta line) is particularly noticeable. As we move outward, the amount of "in situ" stars (green line) rises to a peak around the middle of the bulge-dominated region (closer to the centre). From the location of this peak until the boundary between the bulge and the disc, the fraction of stars coming from the inner regions (red line) increases. Accreted stars in this central region do not show a common behaviour in all the galaxies, but display behaviours ranging from domination of accreted stars (e.g. Ben), to those where the amount of accreted stars is negligible (Helios or Selene).

It is in the disc-dominated region (beyond the bulgedominated part of the galaxy) where discrepancies are larger between type-I, II, and III galaxies. The region of the disc prior to the break radius for type-II galaxies is mainly dominated by stars coming from the inner regions (red line) with the peak located at the break radius or beyond. It is worth noticing that the region where the disc starts to dominate (inner disc, before

\footnotetext{
4 The bulge region is defined along this work as the inner part where the difference between the observed light profile and the best fit of the disc light is higher than $0.1 \mathrm{mag} \operatorname{arcsec}{ }^{-1}$.
}

the break) presents an exodus of stars towards the outer regions (magenta line) that is slightly more evident for galaxies with strong rather than weaker type-II breaks. This exodus of stars plays an important role in shaping type-II breaks (as we demonstrate in Sect. 3.2). Beyond the type-II break, the more important stellar sub-populations that we find are accreted stars (black line) and stars from the inner regions (red line), with the former gaining in importance as we move towards weaker breaks (Castor to Ben) and as we move outwards. All type-II galaxies from RaDES are characterised by an excess of light (type-III secondary break) in the outer parts. The excess of light in these outermost regions is the result of outward motions (red line) and satellite accretion (black line), that is, the accumulation of outward and accreted stars. "In situ" stars (green line) and stars from the outer parts (blue line) are found throughout the disc region prior to the type-III secondary break (more important for strong type-II breaks), with a decrement in the mass fraction of both sub-populations from the position of the type-II break outwards.

However, there is a change in the behaviour of the "in situ" stars (green line) and stars from outer parts (blue line) for type-I and III galaxies with respect to the type-II systems. The fraction of stars in these two sub-populations is lower across the entire disc (after the bulge-dominated region) for type-I and III galaxies than for type-II systems (see Sect. 3.2 where we further quantify this statement). Regarding these two types of galaxies 
(I and III), the main difference displayed between them is found in the radial distribution of accreted stars (black line) and stars coming from the inner parts (red line). At intermediate radii, outward-moving stars (red line) dominate, while at larger galactocentric distances, accreted stars are the main component for type-I galaxies. The importance of accreted stars is higher for type-III galaxies with respect to type-I systems, especially if they display strong type-III breaks (Helios). While type-I and weak type-III galaxies display relatively extended distributions (across tens of kpcs), Helios (a type-III galaxy presenting a strong break) presents a concentration of outward-moving stars right after the bulge dominated region, probably as a consequence of the huge amount of accreted stars in those parts.

The middle-right and right-hand panels (c and d) of Figs. 1 and 2 show the radial (c) and vertical (d) velocity dispersion profiles for the different subpopulations analysed. In this case, we have decided to add the profiles considering all the stellar particles in the simulation (black dashed lines, current locations). It is clear that the inner and outer velocity dispersion profiles display distinct behaviours. All galaxies (regardless of their SB profiles) exhibit the highest values of the velocity dispersion (radial and vertical) in the centre, followed by a radial decrement along the inner regions, that is, from the bulge until a location around the main break.

In contrast, the outer parts may display either a flattening in the dispersion profiles at larger radii or an upturn depending on the galaxy SB profile. Type-II galaxies exhibit outer velocity dispersion profiles for "in situ" stars (green) that are "U-shaped", with the highest values found in the type-III part of the profile and the minimum generally located beyond the type-II break but before the type-III one. The same behaviour is displayed by outward-moving stars (red) and considering all stellar particles together (dashed black line). Similar "U-shaped" profiles are also displayed by the "in situ" subpopulation in the case of type-I galaxies. However, an outer flattening (with a slight increase) is displayed by the outward-moving stars and this shape is also found if we compute the velocity dispersion profiles considering all stellar particles together. On the other hand, outer flattenings or oscillating outer profiles are found for all subpopulations in the case of type-III galaxies starting slightly before the location of the type-III break. We must note that the reported inner decrement in the velocity dispersion profiles of all subpopulations is steeper for stronger type-III breaks. On the other hand, the velocity dispersion profiles displayed by accreted stars is very similar for all galaxies (including types I, II, and III), namely a gradual radial decrement from the highest values (at the centre) to the lowest ones (at around the break location) followed by a flattening or a smoother decrement (in the outskirts). The extension of the region dominated by the gradual decrement for the velocity dispersion of the accreted stars decreases as we move from type-II galaxies to type I and type III.

The fact that the boundary between both regimes (inner and outer behaviour) seems to be located near the break location may suggest a common origin between the light and the velocity dispersion profiles as well as several processes working at once.

\subsection{Interpretation}

These findings can be interpreted in such way that radial redistribution of material (regardless of what physically causes it, including accretion) can explain the different SB profiles. In such a scenario, the presence and strength $(\beta)$ of type-II breaks are a direct consequence of the combination of the radial redistribution of stars and accretion. Stars moving outwards produce a deficit of stars in the disc region (right after the bulge-dominated zone) before the break radius (see magenta line) and, as a consequence, large inner-disc scale-lengths are found in contrast to the outer part (type-II break). As this deficit becomes less important and more stars are found in the region located immediately after the bulge region, shorter disc scale-lengths are displayed, that is, weaker breaks (see trend from Castor to Ben). However, the formation of a type-II break not only depends on the distribution of outward-moving stars; it is the complex combination of this distribution and the distribution of accreted stars that finally shapes type-II galaxies and determines the position of the break. The region where type-II breaks are found and beyond is again dominated by outward-moving stars (solid red line) and accreted stars (black line) that are accumulated in that region. As the fraction of accreted stars is more important at these intermediate galactocentric distances (and also before the type-II break) we detect weaker type-II breaks. Accreted stars smooth out the light profiles giving rise to weak type-II breaks. The secondary type-III breaks that are found in type-II galaxies are basically formed because of the accumulation of accreted and outward-moving (especially accreted) stars in these outer parts which produces an outer excess of light.

Despite the tendency of outward-moving and accreted stars to dominate the mass fraction for all galaxies, it is for type-I and III galaxies where this superiority is even more important. The fact that the fraction of inward-moving and "in situ" stars is lower in the case of type-I and III galaxies makes radial redistribution a more important agent in shaping SB profiles in these galaxies than in type-II galaxies. Therefore, it is the mixing induced by outward-moving and, especially, accreted stars that causes type-II breaks to form pure exponential profiles. The final profile (I or III) will depend on the relative fraction of outwardmoving and accreted stars. As accreted stars start to dominate, especially in the outer parts, type-III breaks appear and become stronger. The overpopulation of outward-moving (red line) stars immediately after the bulge-dominated region found in type-III galaxies with strong breaks (Helios) makes the inner disc scalelength shorter (i.e. a steeper inner light profile).

We must warn the reader here that this interpretation does not necessary establish a time evolution from type-II galaxies to type-III systems. We are only trying to obtain patterns in the radial redistribution of material that can link the different observed SB profiles. Those patterns suggest that radial redistribution of material can change the SB profile of a galaxy from type II, to I, and finally to type III in favourable conditions. However, this interpretation is qualitative and needs some quantification to be more robust. In Sect. 3.2 we quantify some of the previous analysis.

\subsection{Facts favouring this interpretation}

If the previous qualitative reasoning is correct and radial motions do play a role in shaping SB profiles, then Luke (a galaxy displaying a pure exponential light profile) should display a type-III break in the region located between 25 and $35 \mathrm{kpc}$ in to Fig. 2 (upper panel), where accreted stars begin to dominate. The lack of such a break in Luke suggests that, although the radial redistribution of material accumulating in the outer parts in this galaxy is also important, there must be some peculiarities swamping out any possible type-III breaks. In the following we consider all the systems in RaDES and quantify radial redistribution as a function of the break strength $(\beta)$, a continuous quantity, to distinguish between SB types, not focusing on individual galaxies as previously done. This will allow us to further investigate 

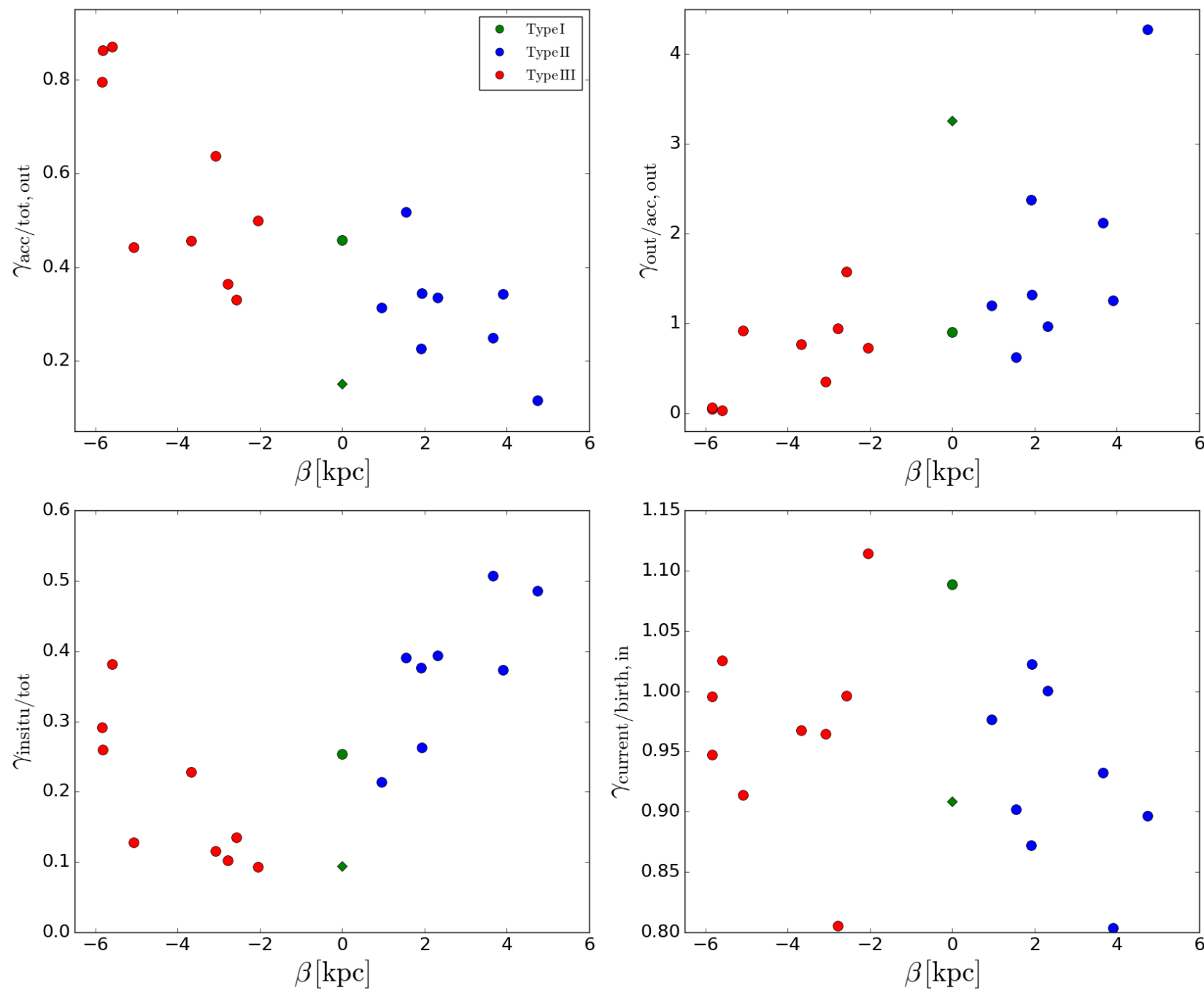

Fig. 3. Quantification of the radial redistribution as a function of the break strength $(\beta)$, defined as $h_{\text {in }}-h_{\text {out }}$. Left-hand, top panel: mass fraction of accreted stars currently located in the outer region with respect to the total stellar mass in that region $\left(\gamma_{\text {acc/totout }}\right)$ as a function of $\beta$. Right-hand, top panel: mass fraction of stars coming from inner radius with respect to the stellar mass of accreted stars currently located in the outer region $\left(\gamma_{\text {out/acc,out }}\right)$ as a function of $\beta$. Left-hand, bottom panel: mass fraction of "in situ" stars in the whole galaxy with respect to the total mass of the galaxy $\left(\gamma_{\text {insitu/tot }}\right)$ as a function of $\beta$. Right-hand, bottom panel: mass fraction of stars currently located in the inner region with respect to the stars born in the same inner region $\left(\gamma_{\text {current/birth,in }}\right)$. Green points represent type-I galaxies, blue points are type-II galaxies and type-III systems are denoted by red points. Zeus is represented by a green diamond as it is a type-I galaxy with signs of ongoing interaction. See text for details.

the reasons for the appearance of type-I galaxies (and other SB profiles) as well as further support our previous interpretation. This analysis of the radial redistribution as a function of $\beta$ allows us to assess the effect of radial redistribution, not only in shaping type-I, II, or III SB profiles, but also in shaping the strength of the different breaks. For simplicity, we focus on two regions that we name the inner region and outer region. The inner region corresponds to the disc-dominated region before the break (type II or III) or before three disc scale-lengths for type-I galaxies. The outer region is the region between the type-II break and the type-III break for type-II galaxies, the region beyond the break for type-III galaxies, and the region beyond three disc scale-lengths for type-I galaxies. We have decided to use three disc scale-lengths as the separation radius as it is there where the breaks are usually found in real galaxies (Pohlen \& Trujillo 2006; Erwin et al. 2008). These two regions are key locations in which we can focus the analysis in order to check the validity of the previous interpretation.
In the left-hand, top panel of Fig. 3 we represent the mass fraction of accreted stars currently located in the outer region with respect to the total stellar mass in the same region $\left(\gamma_{\text {acc/tot,out }}\right)$ as a function of $\beta$. We find a clear correlation with strong type-II galaxies having the lowest values of $\gamma_{\text {acc/tot,out }}$ and strong type-III galaxies displaying the highest ones. In the right-hand, top panel of Fig. 3 we represent the mass fraction of stars coming from inner radius (outward-moving stars) with respect to the stellar mass of accreted stars in the outer region ( $\left.\gamma_{\text {out/acc,out }}\right)$ for the RaDES galaxies. In this case, type-II galaxies with strong breaks display the highest values of $\gamma_{\text {out/acc,out }}$ while type-III galaxies with strong breaks display the lowest ones. The mass fraction of "in situ" stars (along the entire galaxy) with respect to the total galaxy mass $\left(\gamma_{\text {insitu/tot }}\right)$ is represented in the left-hand, bottom panel. In this case we find a "U-shaped" relation between both magnitudes with type-II galaxies displaying the highest values and weak type-III systems showing the lowest. Galaxies with strong type-III breaks also display a good 
amount of "in situ" stars (comparable with systems with weak type-II breaks). Finally, in the right-hand, bottom panel we show the variation of the mass fraction of stars currently located in the inner region with respect to the mass of stars born in the same inner region $\left(\gamma_{\text {current/birth,in }}\right)$ as a function of $\beta$. Type-II galaxies exhibit an interesting correlation with some scatter in which strong type-II breaks display low values of $\gamma_{\text {current/birth,in }}$ and weak type-II breaks or even type-I systems present higher values.

The four correlations support the proposed scenario in which different degrees of radial redistribution of material give rise to the different observed SB profiles. The correlation found for $\gamma_{\text {acc/tot,out }}$ reflects that accretion is more important in type-I and III galaxies than in type-II galaxies, confirming that accretion can convert type-II breaks into pure exponentials or even type-III galaxies. The behaviour of $\gamma_{\text {out/acc,out }}$ with type-II galaxies displaying larger values reflects again that accreted stars are less abundant in the outer region for these galaxies (causing outer disc scale-length to be shorter) and that outward-moving stars accumulate around the type-II breaks and beyond. The shape of the $\gamma_{\text {insitu/tot }}$ versus $\beta$ relation allows us to confirm that the amount of "in situ" stars for type-II galaxies is higher than for type-I or III galaxies, verifying our interpretation that radial redistribution is less important in type-II galaxies. However, the three type-III galaxies with the strongest breaks invert this trend and display similar values of weak type-II discs. It is the vast amount of accreted stars in these systems (see the upper lefthand panel of Fig. 3) that causes them to have such strong type-III breaks despite the quantity of "in situ" stars. It is the correlation of $\gamma_{\text {current/birth,in }}$ with $\beta$ for type-II galaxies that leads us to the conclusion that one of the main factors shaping type-II breaks is the exodus of stars from inner regions. The lack of stars in the inner region produces a shallower inner light profile which strengthens the break. The fact that this behaviour is not found for type-III galaxies reinforces the interpretation provided in this work.

It is worth noticing that the importance of all the processes described is always intermediate for type-I galaxies with respect to the other two types. This allows us to conclude that type-I galaxies are "boundary" galaxies with properties in between those of type-II and type-III galaxies and that they exhibit small peculiarities (that need to be further analysed) swamping out any possible breaks. As the reader may have noticed, in the four correlations there is always one type-I galaxy slightly off the correlation, Zeus (represented in Fig. 3 with green diamonds). Zeus still has a satellite companion and thus is not the best example for this analysis. The presence of the companion in Zeus explains its position out of the correlation in all the relations (see Few et al. 2012, for further information on Zeus).

Returning to Luke, the lack of a type-III break can be explained based on the radial redistribution of material in these outer regions. Although accreted stars dominate with respect to the other subpopulations (black line), it is not as important in absolute terms as for the other type-III galaxies (see top-right panel of Fig. 3). In addition, there is a clear negative radial trend of the stars coming from the inner regions that end up at such galactocentric regions (red line). Both facts, along with the exodus of stars located at those external parts and that have moved outwards (magenta line), mean that stars do not accumulate in the outskirts, preventing any type-III break from being observed.

\subsection{On the behaviour of the velocity dispersion radial profiles}

Previous works have found that type-III profiles come from a kinematically hot component. Herpich et al. (2017) suggested that a combination of a strong bar with a low initial halo spin is able to make stars migrate outward to form type-III breaks that are populated by stars that acquire high-velocity dispersions in the migration process. In addition, another way to inhabit the outer parts of type-II galaxies with a hot component was found by Minchev et al. (2012) via smooth accretion of gas. In that work, this accretion leads to the formation of outer type-III breaks coexisting with the former downbending profile. The resemblance of their type-II + type-III combined profiles to the ones shown in this work is remarkable. However, while Minchev et al. (2012) found a clear correlation between the location of this secondary type-III break and an upturn in the stellar radial velocity dispersion (see their Fig. 17), we do not find such clear relation. We obtain that the stellar velocity dispersion profiles of the type-II RaDES galaxies display upturns starting at intermediate locations between the inner type-II break and the outer type-III one (e.g. Castor) or related with the location of the primary type-II break (e.g. Selene and Ben). The discrepancies among both works are understandable considering the differences in the simulation recipes as well as the fact that the Minchev et al. (2012) analyses do not include stellar accretion. In fact, the location of the upturn in velocity dispersion seems to be related (in all cases) to the position where the mass fraction of accreted stars (see panel b) starts to increase, suggesting that accreted stars have a greater impact on the global velocity dispersion than on the light profile. However, not only do the "global" radial and vertical velocity dispersion profiles (considering all particles, including accretion) show this upturn, but so do other subpopulations of non-accreted stars (see Figs. 1 and 2). Regarding pure type-III galaxies, they do not display this velocity dispersion upturns at all. In contrast, they mainly show flattenings that start generally before the type-III breaks, again related to the radial position where the accreted stars begin to dominate.

To sum up, we can claim that all the RaDES galaxies display stellar velocity dispersions in the outer parts that are higher than that expected from the extrapolation of the behaviour in the inner parts (before the regions where the breaks appear), although only type-I and II galaxies exhibit clear upturns that might be related to the accretion.

\section{Effect of radial redistribution in shaping stellar population age and metallicity profiles}

According to the previous section, radial redistribution of stars seems to play an important role in shaping the different observed SB profiles. However, from an observational point of view, this statement is difficult to confirm and might fall into the box of speculative theories. To solve this issue, other observables have to be analysed in order to provide this hypothesis with predictions that can be tested. Some of the observables that can be computed using modern observing techniques are stellar age and metallicity profiles, especially in the inner regions where the signal is higher. Ruiz-Lara et al. (2017, hereafter TRL17) recently studied such profiles using the spectroscopic data provided by the CALIFA survey (Sánchez et al. 2012). The authors, analysing a sample of 214 spiral galaxies, found that type-II systems tend to display steeper stellar age and metallicity profiles than type-I and III galaxies, with the latter showing the shallowest ones. They interpret those findings as a consequence of radial 
T. Ruiz-Lara et al.: Shaping surface brightness profiles via stellar radial motions
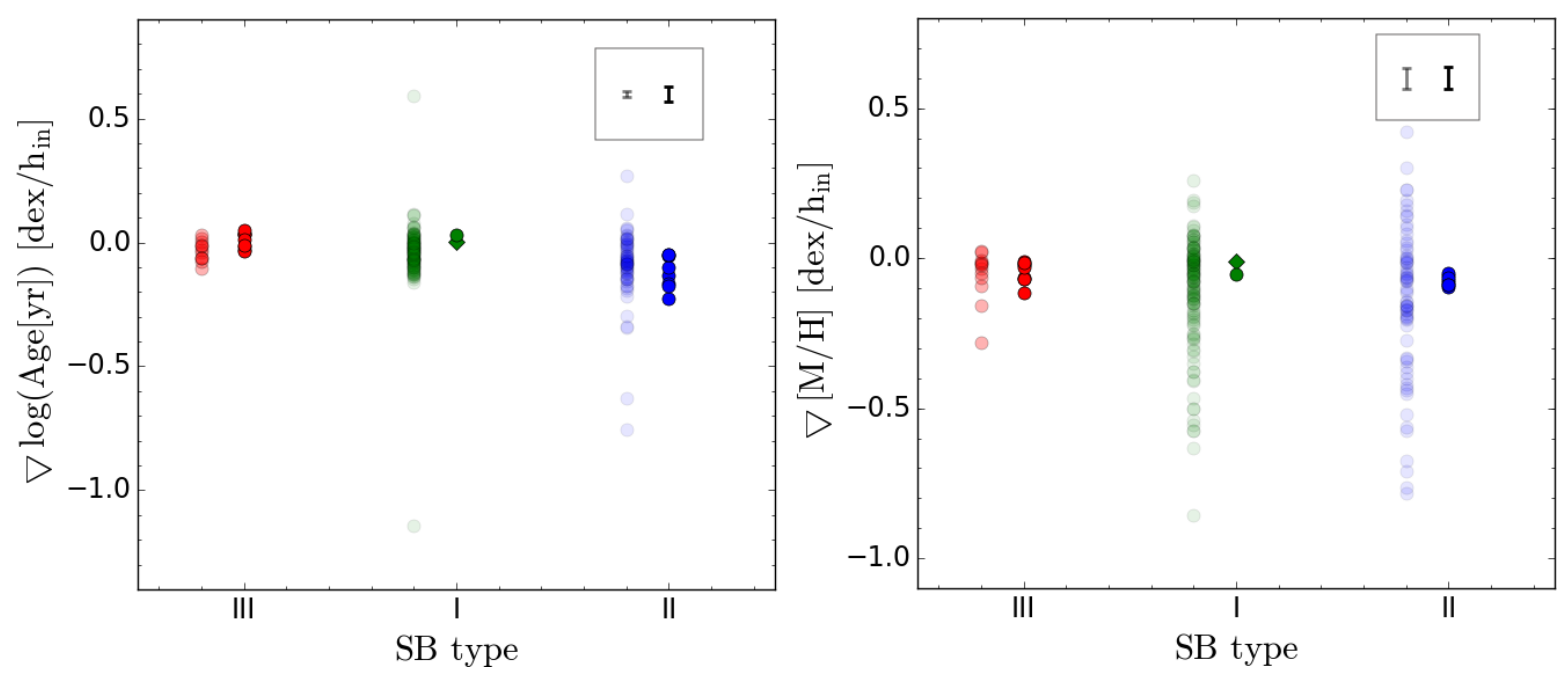

Fig. 4. Comparison of the distributions of the inner gradients of the stellar age (left-hand panel) and metallicity (right-hand panel) profiles for type-I (green), II (blue), and III (red) galaxies from RaDES with the CALIFA mass-weighted distributions (TRL17, symbols with transparency). Zeus is again represented by a green diamond (type-I galaxy with signs of ongoing interaction). Typical errors in the gradient values for the observations and the simulations are shown with the black error bars within the boxes (left, typical errors in the CALIFA values; right, typical errors in the case of the gradients from the simulations).

Table 2. Error-weighted average and dispersion values of the inner gradients of the stellar age and metallicity (see text for details) for the RaDES set of galaxies.

\begin{tabular}{lccc}
\hline \hline & \multicolumn{3}{c}{ SB profile } \\
\hline & Type III & Type I & Type II \\
$\nabla \log ($ Age $[\mathrm{yr}])\left[\mathrm{dex} / h_{\text {in }}\right]$ & $0.02(0.03)$ & $0.004(0.01)$ & $-0.09(0.06)$ \\
$\nabla[\mathrm{M} / \mathrm{H}]\left[\mathrm{dex} / h_{\text {in }}\right]$ & $-0.05(0.03)$ & $-0.02(0.02)$ & $-0.07(0.02)$ \\
\hline
\end{tabular}

Notes. Units are in dex $/ h_{\text {in }}$.

redistribution of material being more effective in type-III galaxies than in type-I or II systems, in agreement with the scenario proposed in this work.

In order to compare with those observational results, we compute mass-weighted age and metallicity profiles for all the RaDES galaxies by averaging all disc particles using $0.5 \mathrm{kpc}$-wide radial bins (see Ruiz-Lara et al. 2016a). Afterwards, we perform error-weighted linear fits to those profiles in the inner and the outer regions, separately. We define these inner and outer regions following TRL17 prescription and matching the inner and outer regions defined previously (see Sect. 3.2). We decided to perform this analysis only in the inner parts of the profiles as in the analysis presented in TRL17. In Fig. 4 we show the distributions of the inner gradients for the stellar age (left-hand panel) and metallicity (righthand panel) profiles distinguishing between type-I (green), II (blue), and III (red) galaxies over-plotting the results coming from the CALIFA observations (TRL17, symbols with transparency). Table 2 shows the error-weighted average and dispersion values of the inner gradients of the stellar age and metallicity for the type-I, II, and III galaxies from RaDES.

The values of the age and metallicity gradients for individual galaxies from the simulations are consistent with the values presented in TRL17 for the CALIFA sample (see Fig. 4). The low number of simulated galaxies with respect to the observed ones hampers a complete comparison of both distributions, however, some similarities can be highlighted. Type-II galaxies display a larger dispersion in age gradient for the simulations and the observations, while in the case of metallicity gradients, the dispersions are compatible for the three types of galaxies. TypeII galaxies present the steepest average gradients for the age $\left(-0.09 \mathrm{dex} / h_{\text {in }}\right)$ and the metallicity $\left(-0.07 \mathrm{dex} / h_{\text {in }}^{-1}\right)$. On the other hand, type-I systems show the shallowest profiles $(0.004$ and $-0.02 \mathrm{dex} / h_{\text {in }}$ for age and metallicity, respectively) with type-III systems displaying intermediate values (or even positive values in the case of the age: 0.02 and $-0.05 \mathrm{dex} / h_{\text {in }}$, for the age and metallicity gradients, respectively). In TRL17, type-III systems are found to have the shallowest profiles with a clear trend from type II (steepest) to type I (intermediate) and type III. The differences between the simulated values presented in this work and the observational results from CALIFA are reasonable and easily explained considering the low number of simulated systems analysed. However, the similarities and the consistency between the gradients for individual galaxies are reassuring.

In Fig. 5 we study the dependency of the stellar age and metallicity gradients displayed by the RaDES galaxies as a function of the strength of their breaks $(\beta)$. If radial redistribution of material does play an important role in shaping SB profiles (see Sect. 3.1) and it also affects age or metallicity gradients of the inner disc (see Fig. 4), then there should be some kind of correlation between these gradients and the detailed shape of the break. In fact, this is what we show in Fig. 5. Type-II galaxies with strong breaks (less influenced by radial redistribution, especially accretion) display steeper stellar age and metallicity profiles than type-II systems with weak breaks or even type-I galaxies. A consistent behaviour is also found in the case of the age profiles for type-III galaxies in which systems with weak breaks (less affected by accretion) display steeper profiles than galaxies 

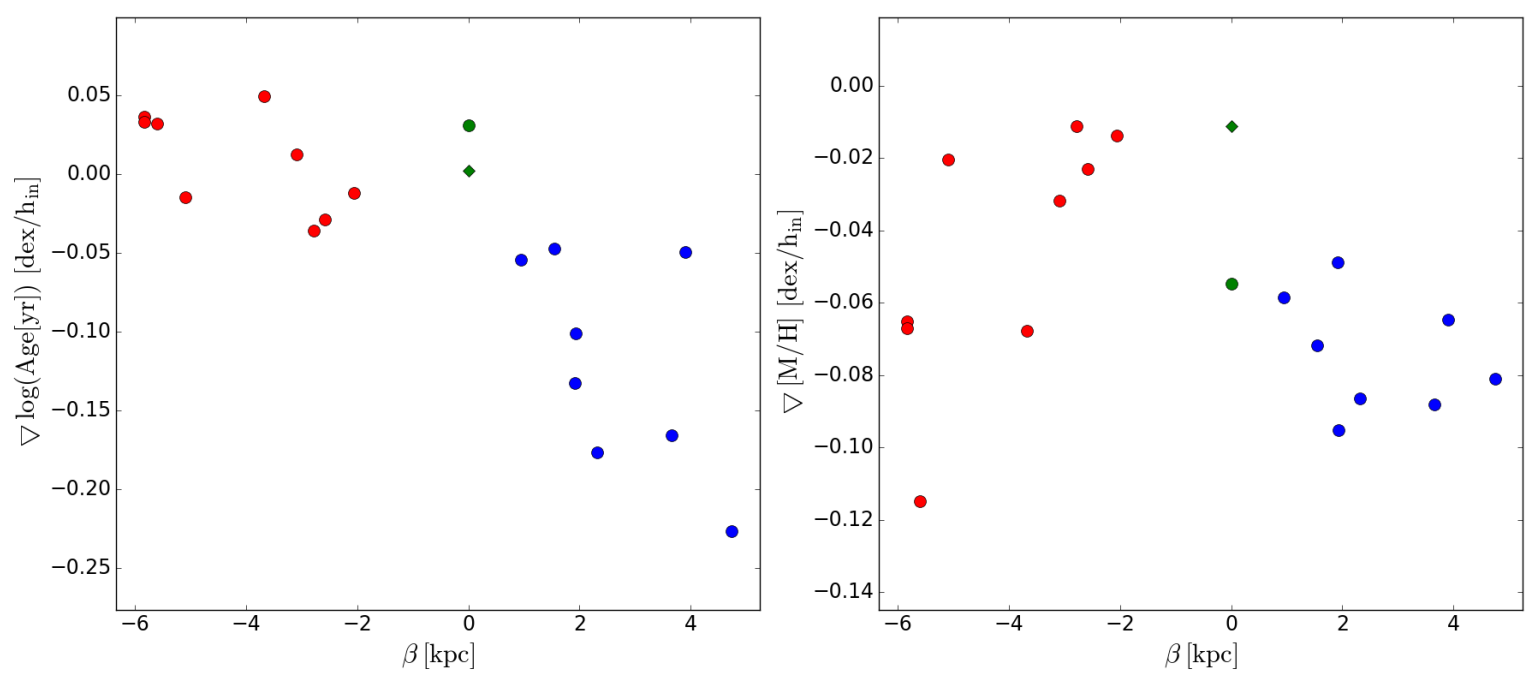

Fig. 5. Stellar age (left-hand panel) and metallicity (right-hand panel) inner gradients as a function of the break strength $(\beta)$ for the RaDES set of galaxies. The gradients for type-I, II, and III galaxies are indicated in green, blue, and red, respectively. Zeus is again represented by a green diamond (type-I galaxy with signs of ongoing interaction).
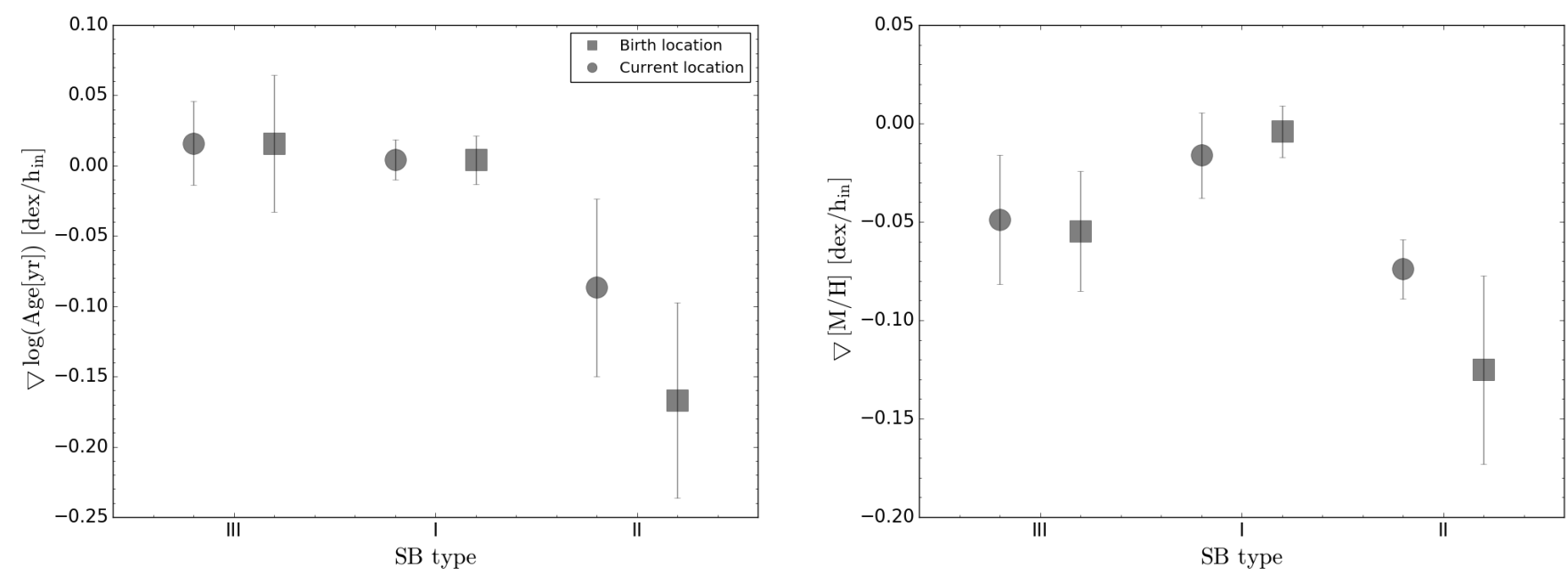

Fig. 6. Distribution of the inner gradients of the stellar age (left-hand panel) and metallicity (right-hand panel) profiles for type-I, II, and III galaxies considering current locations (grey circles) or birth locations (grey squares) of the stars to compute the stellar age and metallicity profiles.

with strong breaks; in which case, even positive gradients are displayed. However, in the case of the metallicity gradients, this trend is inverted. Type-III galaxies with weak breaks have shallower profiles than systems with strong breaks (which are more affected by accretion). This inverted trend can be explained by the characteristics of the accreted stars. In Ruiz-Lara et al. (2016a) we showed that accreted stars populating the RaDES galaxies are mainly old, metal-poor stars. As a consequence, the accumulation of accreted stars in the outer parts of type-III galaxies giving rise to these kind of systems causes the outer parts to be old (positive age gradients) and relatively metal-poor (producing steeper negative profiles). Based on Fig. 5 we can conclude that, not only does radial redistribution of material affect the shape of observed light distributions, but also accretion has to play a key role in shaping observed stellar age and metallicity profiles.

All these results and the similarities between the observational results (TRL17) and the theoretical work presented in this paper seem to indicate that radial redistribution of material and accretion shape the SB profiles and generally flatten the age and the metallicity profiles with the exception of the metallicity profiles for type-III galaxies due to the accumulation of metal-poor, accreted stars in the outer disc. The greater the efficiency of this redistribution, the larger the flattening effect. As a consequence, we would expect steeper age and metallicity gradients at birth than what we observe presently due to the effect of the radial redistribution of material. To shed light on this aspect and the true role of this redistribution in shaping the age and metallicity profiles we have also analysed the inner gradients of these stellar parameters in the absence of radial motions, that is, considering birth locations (see Fig. 6).

Figure 6 shows the same information as Fig. 4 but focusing on the effect of radial motion of stars on the stellar age and metallicity gradients. In this case we show mean values for type-I, II and III systems, not the values for all the individual galaxies. Grey circles represent the mean values of the stellar age and metallicity gradients (averaging among SB types) considering stars located at their current locations. Grey squares represent the same mean quantities but this time computed using stars located at their birth locations (thus avoiding the effect of radial 
motions). Strikingly, the inner stellar parameter gradients for type-I and III galaxies do not seem to be especially affected by radial redistribution of material despite steeper gradients being expected when considering birth locations and shallower ones at the current locations. The case of type-II galaxies however shows that the migration of stars flattens the stellar profiles considerably. The fact that, even at birth locations type-I and III galaxies seem to display shallow age and metallicity profiles (and definitely shallower than type-II galaxies), suggests that the differences found in Fig. 4 must have been imprinted at birth. This points toward the existence of formation mechanisms producing steep stellar age and metallicity profiles for type-II galaxies and shallow profiles for type-I and III systems. These findings merit further investigation focusing on the early stages of the formation of these systems, a study beyond the scope of this paper, focused on the relation between radial redistribution and the shape of the SB profiles.

\section{Conclusions}

In this work we propose a scenario in which radial redistribution of stars can significantly affect the light distributions shaping observed SB profiles. Redistribution of material, especially outward-moving and accreted stars, is important in all RaDES galaxies regardless of their SB profile. The accretion and migration of stars particularly prevails in type-I and III galaxies while the fraction of "in situ" stars is slightly higher in type-II systems. Both aspects suggest that radial redistribution is less significant in systems with downbending light profiles. Outward-moving stars from the region just beyond the bulge produce extended inner light profiles and the accumulation of these and accreted stars determines the presence and strength of type-II breaks. An increase in the fraction of accreted stars causes the fading of the type-II breaks into pure exponentials and even the shaping of type-III breaks whose strength depends on the number of accreted stars settled in the outer parts. We have proven that type-I galaxies present properties that lie between those of type-II and type-III galaxies. In addition, the stars populating the locations where breaks are found and beyond (where accreted stars begin to dominate) present higher velocity dispersions than what is expected by extrapolating the behaviour in the inner parts in agreement with previous works (e.g. Minchev et al. 2012; Herpich et al. 2017). This scenario seems to leave signatures in the radial distribution of stellar properties such as age and metallicity, especially due to the effect of accreted stars. Shallower inner profiles are found in galaxies where radial redistribution is more important (type I and III) while type-II galaxies present steeper inner gradients. These signatures have been observed recently in a sample of nearby galaxies from the CALIFA survey (Ruiz-Lara et al. 2017). However, these differences in the inner gradients of stellar population properties can also be found in the absence of stellar radial motion suggesting that they may be imprinted at birth. Further work merging observational analysis with realistic cosmological simulations ${ }^{5}$ in a larger number of systems (to improve the statistics) is needed to properly understand how the different SB profiles come into shape.

Acknowledgements. We thank the referee for very useful suggestions and comments that have helped improve the current version of this manuscript. This research has been partly supported by the Spanish Ministry of Science and

\footnotetext{
5 Future analyses will aim to identify star particles more rigorously according to their observational characteristics, as proposed in Thompson et al. (2018).
}

Innovation (MICINN) under grants AYA2014-53506-P and AYA2014-56795-P; and by the Junta de Andalucía (FQM-108). We acknowledge the generous allocation of resources from the Partnership for Advanced Computing in Europe (PRACE) via the DEISA Extreme Computing Initiative (PRACE-3IP Project RI312763 and PRACE-4IP Project 653838), STFC's DiRAC Facility (COSMOS: Galactic Archaeology - ST/J005673/1, ST/H008586/1, ST/K00333X/1), and the University of Hull's High Performance Computing Facility (VIPER). TRL thanks the support of the Spanish Ministerio de Educación, Cultura y Deporte by means of the FPU fellowship. CGF acknowledges support from the FP7 European Research Council Starting Grant LOCALSTAR.

\section{References}

Abadi, M. G., Navarro, J. F., Steinmetz, M., \& Eke, V. R. 2003, ApJ, 591, 499 Bakos, J., Trujillo, I., \& Pohlen, M. 2008, ApJ, 683, L103

Bernard, E. J., Ferguson, A. M. N., Barker, M. K., et al. 2012, MNRAS, 420, 2625

Bernard, E. J., Ferguson, A. M. N., Richardson, J. C., et al. 2015, MNRAS, 446, 2789

Bird, J. C., Kazantzidis, S., \& Weinberg, D. H. 2012, MNRAS, 420, 913

Bland-Hawthorn, J., Vlajić, M., Freeman, K. C., \& Draine, B. T. 2005, ApJ, 629, 239

Borlaff, A., Eliche-Moral, M. C., Beckman, J., \& Font, J. 2016, A\&A, 591, L7

Elmegreen, B. G., \& Struck, C. 2016, ApJ, 830, 115

Erwin, P., Beckman, J. E., \& Pohlen, M. 2005, ApJ, 626, L81

Erwin, P., Pohlen, M., \& Beckman, J. E. 2008, AJ, 135, 20

Few, C. G., Gibson, B. K., Courty, S., et al. 2012, A\&A, 547, A63

Florido, E., Battaner, E., Guijarro, A., Garzón, F., \& Castillo-Morales, A. 2006, A\&A, 455, 467

Freeman, K. C. 1970, ApJ, 160, 811

Gibson, B. K., Courty, S., Cunnama, D., \& Mollá, M. 2013, Asociacion Argentina de Astronomia La Plata Argentina Book Series, 4, 57

Gogarten, S. M., Dalcanton, J. J., Williams, B. F., et al. 2010, ApJ, 712, 858

Grand, R. J. J., Springel, V., Kawata, D., et al. 2016, MNRAS, 460, L94

Herpich, J., Stinson, G. S., Dutton, A. A., et al. 2015, MNRAS, 448, L99

Herpich, J., Stinson, G. S., Rix, H.-W., Martig, M., \& Dutton, A. A. 2017, MNRAS, 470, 4941

Jonsson, P. 2006, MNRAS, 372, 2

Leitherer, C., Schaerer, D., Goldader, J. D., et al. 1999, ApJS, 123, 3

Martín-Navarro, I., Bakos, J., Trujillo, I., et al. 2012, MNRAS, 427, 1102

Martínez-Serrano, F. J., Serna, A., Doménech-Moral, M., \& DomínguezTenreiro, R. 2009, ApJ, 705, L133

Minchev, I., \& Famaey, B. 2010, ApJ, 722, 112

Minchev, I., Famaey, B., Quillen, A. C., et al. 2012, A\&A, 548, A126

Patterson, F. S. 1940, Harvard College Observatory Bulletin, 914, 9

Pérez, I. 2004, A\&A, 427, L17

Pilkington, K., Few, C. G., Gibson, B. K., et al. 2012, A\&A, 540, A56

Pohlen, M., \& Trujillo, I. 2006, A\&A, 454, 759

Pohlen, M., Dettmar, R.-J., Lütticke, R., \& Aronica, G. 2002, A\&A, 392, 807

Quillen, A. C., Minchev, I., Bland-Hawthorn, J., \& Haywood, M. 2009, MNRAS, 397, 1599

Roediger, J. C., Courteau, S., Sánchez-Blázquez, P., \& McDonald, M. 2012, ApJ, 758,41

Roškar, R., Debattista, V. P., Quinn, T. R., Stinson, G. S., \& Wadsley, J. 2008a, ApJ, 684, L79

Roškar, R., Debattista, V. P., Stinson, G. S., et al. 2008b, ApJ, 675, L65

Ruiz-Lara, T., Few, C. G., Gibson, B. K., et al. 2016a, A\&A, 586, A112

Ruiz-Lara, T., Pérez, I., Florido, E., et al. 2016b, MNRAS, 456, L35

Ruiz-Lara, T., Pérez, I., Florido, E., et al. 2017, A\&A, 604, A4

Sánchez, S. F., Kennicutt, R. C., Gil de Paz, A., et al. 2012, A\&A, 538, A8

Sánchez-Blázquez, P., Courty, S., Gibson, B. K., \& Brook, C. B. 2009, MNRAS, 398, 591

Sánchez-Menguiano, L., Sánchez, S. F., Kawata, D., et al. 2016, ApJ, 830, L40

Sellwood, J. A., \& Binney, J. J. 2002, MNRAS, 336, 785

Steinmetz, M., \& Navarro, J. F. 2002, New Astron., 7, 155

Teyssier, R. 2002, A\&A, 385, 337

Thompson, B. B., Few, C. G., Bergemann, M., et al. 2018, MNRAS, 473, 185

Trujillo, I., \& Pohlen, M. 2005, ApJ, 630, L17

van der Kruit, P. C. 1987, A\&A, 173, 59

White, S. D. M., \& Rees, M. J. 1978, MNRAS, 183, 341

Yoachim, P., Roškar, R., \& Debattista, V. P. 2012, ApJ, 752, 97

Younger, J. D., Cox, T. J., Seth, A. C., \& Hernquist, L. 2007, ApJ, 670, 269 